5 Abstract penetration.

\title{
SOIL-PROJECTILE INTERACTIONS DURING LOW VELOCITY PENETRATION
}

\author{
Mehdi Omidvar, Magued Iskander ${ }^{\ddagger}$, Stephan Bless \\ New York University, USA
}

Results of penetration tests into refractive index matched granular media are presented in this study. These tests, performed at velocities of approximately $20 \mathrm{~m} / \mathrm{s}$, demonstrate the use of transparent soils in visualizing insitu granular kinematics, non-intrusively, during dynamic penetration in granular media. The transparent soil surrogate described herein has macroscopic physical and mechanical properties that mimic the behavior of natural soils. Digital image correlation was used to obtain soil displacements and corresponding granular kinematics from the acquired images. An analysis of measured deformations at the meso scale is presented to investigate various features of soil-projectile interaction during

Keywords: Transparent soil; granular media; index matching; projectile; rod; visualization; interaction; shear; dilation

\footnotetext{
‡ Professor and Chair, Civil \& Urban Engineering Department, Polytechnic School of Engineering, New York University. Six Metrotech Center, Brooklyn, NY 11201. Tel: (718) 260-3016, Fax: (718) 260-3433, Email: Iskander@nyu.edu (Corresponding Author).
} 


\section{Introduction}

The study of impact and penetration into granular media has received a surge of interest in the past two decades. Earlier studies have been motivated by military applications, while more recently, a combination of military and civilian applications have sparked interest in the subject. Prediction of penetration has been made possible in the past by means of empirical relationships derived mainly from global observations of penetration characteristics such as impact velocity and terminal penetration depth. Phenomenological models based on physics principles and inspired by observations of deceleration after impact have further strengthened our understanding of penetration dynamics (Omidvar et al. 2014a). Experiments designed to provide data for development of predictive tools have been limited, in the past, to these global observations.

In recent years, significant advances in computational resources and numerical modeling capabilities have made possible modeling of complex phenomena and interactions during rapid penetration in granular media. Increasingly, these numerical predictive tools can provide information at finer length scales. Global observations of penetration, while useful, have limited utility in development and validation of advanced predictive models. The demand for information at finer length scales has led to the development of novel and innovative techniques to visualize penetration at meso and micro scales (e.g., Collins et al. 2011; Borg et al. 2013; Nordstrom et al. 2014). In this paper, visualization of rapid penetration at a meso scale is made possible using transparent soils consisting of frictional soil surrogates with bulk physical and mechanical properties similar to those of natural soils.

In the next sections, first a background is provided on transparent soils used in this study. The experimental setup used to visualize deformations during rapid penetration into a transparent soil target is then briefly described, including the optical setup, projectile accelerator, sample preparation, and image analysis methods used to obtain soil displacements from acquired images. Finally, a deformation analysis is presented to study various features of soil-projectile interaction during penetration, at the meso scale. 


\section{Experimental setup}

\subsection{Transparent soil to model sand}

Transparent soils are made by saturating the pores of a transparent granular soil surrogates having a matched optical refractive index. Several different families of transparent soils have been identified in the literature for simulating natural granular media. A range of problems mainly dealing with soil-structure interactions have been studied using transparent soils (Iskander 2010). The goal of employing transparent soils in subscale tests is to visualize internal kinematics. As such, an important step in adopting a suitable transparent granular material is to investigate the extent to which the physical and mechanical properties of the soil surrogate mimic those of natural soils. Several soil surrogates have been employed in the literature, including crushed silica gel and silica gel beads, amorphous silica powder, glass beads, crushed fused quartz (Sadek et al 2002, Iskander et al 2002, 2003). Among the materials available, fused quartz exhibits properties most similar to natural angular sand. Therefore, fused quartz was adopted in the present study. Mechanical properties of crushed fused quartz can be found in Ezzein and Bathurst (2011) and Guzman et al (2014).

The size distribution of the fused quartz particles used in this study are shown in Fig 1, from which the mean mass diameter $\left(d_{50}\right)$ of the particles was found to be $580 \mu \mathrm{m}$. Particle shapes were analyzed using a dynamic imaging particle analyzer (Sympatec QicPic). Results of the particle analysis, shown in the inset of Fig 1 reveal that crushed fused quartz is comprised of angular shaped particles having mean sphericity and aspect ratio of 0.83 and 0.57, respectively. Prior to sample preparation, crushed fused quartz was sieved to produce a narrower size distribution in the range of $400-700 \mu \mathrm{m}$, with a d50 of $450 \mu \mathrm{m}$, to enhance transparency. Transparent samples were made by pluviating crushed fused quartz into a chamber containing index-matched pore fluid. The pore fluid was a blend of two mineral oils, Puretol 7 and Krystol 40, with RI of 1.4635 and 1.4505 , respectively, at $22{ }^{\circ} \mathrm{C}$. The two oils were mixed in appropriate proportions to produce an RI of 1.4585 , which is the reported RI of fused quartz.

In order to visualize penetration into the transparent granular media, a technique known as the embedded plane technique was used, whereby a mono layer of opaque particles were physically seeded within a plane midway into the transparent soil target. The opaque particles consisted of dyed crushed fused quartz. The test chamber measured 480 by 180 by 


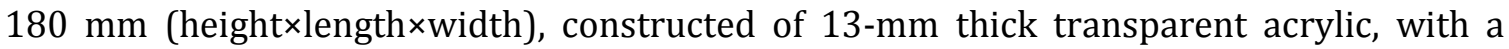
detachable lateral wall to allow for lateral pluviation of the fused quartz. Samples were prepared in three general steps. First, the chamber was placed laterally, and fused quartz was pluviated to mid depth of the target. Next, a mono layer of dyed crushed fused quartz was pluviated on the surface. Finally, the remaining half of the target was pluviated and the chamber was placed upright for testing. Further details of sample preparation can be found in Omidvar et al. (2015). Three samples were prepared in this study.

The mass of the oven-dried fused quartz pluviated as well as that of the embedded plane particles was recorded during sample preparation in order to determine the dry density of the sample. Pluviation through the pore fluid invariably resulted in a loose packing. The sample was allowed to age for a period of $72 \mathrm{~h}$, at which point the relative density of the sample had increased by approximately $10 \%$ due to self compaction. Samples had dry densities in the range of $1.11-1.17 \pm 0.02 \mathrm{~g} / \mathrm{cm} 3$, corresponding to a relative density of 45 $63 \%$.

\subsection{Optical setup}

Transparent samples were illuminated during testing, and a high-speed camera was used to acquire images for subsequent analysis. The setup is schematically shown in Fig 2 . Controlled indirect lighting was used to illuminate the sample. Several considerations influenced the design of the illumination system, including elimination of flicker, light intensity requirements, uniform illumination requirements, and temperature sensitivity of transparent soils, as described in Omidvar et al. (2015). A set of two $500 \mathrm{~W}$ tungsten halogen light sources was used to illuminate the sample from the side opposite to the camera, with an additional $250 \mathrm{~W}$ light source used for alignment. Indirect lighting was used; the lights were aimed at a large piece of white foam core placed behind the sample, which provided Lambertian reflectance.

A NAC HX-5 high-resolution CMOS camera was employed for image acquisition. The camera was equipped with a Nikkor 1.2 f-stop aperture lens. Digital 24-bit monochrome images were acquired for image analysis. An imaging frequency of $6 \mathrm{kHz}$ and an exposure time of $50 \mu$ s were used in this study, which permitted an image resolution of $1152 \times 896$ pixels. A high precision graduated fiducial marker was used to determine the camera magnification. The optical setup resulted in a magnification in the range of $6.11-7.15$ pixels $/ \mathrm{mm}$, resulting in a spatial resolution of approximately $140-164 \mu \mathrm{m}$. 


\subsection{Electro-pneumatic accelerator and projectiles tested}

The accelerator consisted of an electro-pneumatic launcher, with pressurized helium used to provide energy to propel the projectile, and an electro-pneumatically operated solenoid valve used to deliver energy to the projectile. Further details of the launcher design and performance can be found in Cave et al. (2014). The projectiles used in the tests consisted of cylindrical rods with conical and blunt nose shapes, having a mass of 33.44 and $40.05 \mathrm{~g}$, respectively. Both projectiles had a diameter of $10 \mathrm{~mm}$. The stability of the penetrators during penetration was promoted by moving the center of mass of the penetrator closer to its center of pressure, that is, the point of action of the sum of forces on the penetrator. This was done by boring a hole in the rear end of the projectiles to a depth of $63 \mathrm{~mm}$. An additional test was performed under quasi-static loading to visualize the qualitative and quantitative differences in soil-projectile interactions under dynamic and quasi-static penetration. The quasi-static penetration test was performed in samples prepared following procedures outlined above. A hydraulic actuator was used to push a long rod having the same cross-sectional area as the projectiles used in dynamic penetration tests. The long rods were jacked into the transparent soil sample at a constant rate of $0.1 \mathrm{~mm} / \mathrm{s}$. Penetration resistance was recorded using a load cell attached to the penetrator.

\section{Image processing and kinematic analysis tools}

Images acquired from the high-speed camera during penetration into the transparent soil samples were used to perform kinematic analysis. The analysis tools included digital image correlation (DIC), algorithms to compute shear and volumetric strains, and an algorithm to derive cumulative Lagrangian trajectories from the incremental Eulerian DIC analyses. These tools are briefly described next.

\subsubsection{Digital image correlation}

DIC was performed on successive image pairs obtained from the high-speed camera recordings, to obtain the relative displacement fields between the images. DIC has been extensively used in the study of surface displacements (e.g., Liu and Iskander 2004; Hild and Roux 2006; Sutton et al 2009; and Pan 2009). The general steps involved in DIC analysis are schematically shown in Fig 3. Central to success of DIC analysis is the selection of the optimization criterion. The criterion used in this study was a zero-mean normalized sum of 
squared difference (ZNSSD) criterion, which was performed by minimizing the sum of the square of the difference between corresponding interrogation windows, as follows:

$$
\operatorname{ZNSSD}(m, n)=\left[\frac{\sum_{i=0}^{M-1} \sum_{j=0}^{N-1}[f(i, j)-\bar{f}]}{\sqrt{\sum_{i=0}^{M-1} \sum_{j=0}^{N-1}[f(i, j)-\bar{f}]^{2}}}-\frac{\sum_{i=0}^{M-1} \sum_{j=0}^{N-1}[g(i+m, j+n)-\bar{g}]}{\sqrt{\sum_{m=0}^{M-1} \sum_{n=0}^{N-1}[g(i, j)-\bar{g}]^{2}}}\right]^{2}
$$

ZNSSD optimization algorithms often operate in a local range of pixels, by calculation of Eq. (1) within a region spanning the search area, searching for the minimum ZNSSD. The correlation involves subtracting a local mean of pixel intensities, as well as normalization by the standard deviation. This results in a correlation that is insensitive to local linear changes in illumination (Tong 2005).

Further enhancements were made in the image analysis to improve the image correlations, consisting of (1) multi-grid interrogation, also known as window deformation (Westerweel et al. 1997), and (2) sub-pixel estimation. An initial window size of $64 \times 64$ pixels was selected for the analysis, with a final window size of $32 \times 32$ pixels, and a $50 \%$ overlap of interrogation windows. The selection of these parameters resulted in calculation of displacement fields on a 16 pixel square grid, corresponding to a displacement vector at approximately every $4.5 \mathrm{D}_{50}$.

\subsubsection{Strain calculation}

Shear strains were calculated following methods described by Omidvar et al. (2014b). In summary, displacement vectors obtained from conventional DIC analysis can be used to generate strain using continuum mechanics definitions of infinitesimal or finite strain. In the presence of large deformations, the finite strain theory must be resorted to (e.g., Lubliner 2008). A finite strain formulation was employed in this study to calculate strain and rotations from DIC displacement fields. To this end, the image field of view was first discretized into triangular elements, by connecting neighboring center points of the interrogation windows. Discretization may be achieved using a number of algorithms, the majority of which have been derived for finite element analysis. In this study, Delaunay triangulation (de Berg et al. 2008) was employed, which is based on connecting discrete points into a triangular mesh such that the circumcircle of each triangle does not contain points from any other triangle. 
Upon discretization into triangular elements, strain components are calculated for each element using nodal displacements obtained from the DIC analysis. Since each node of a triangle corresponds to a center of an interrogation window, horizontal and vertical displacement components of each node is available from the DIC analysis. A simple means of calculating strains from nodal displacements is to employ shape functions used in finite element analysis. In this study, constant strain triangles were employed, resulting in linear shape functions. Logarithmic shear strains were also employed because of the large strains encountered during projectile penetration.

\subsubsection{Lagrangian trajectories from incremental DIC analysis}

In order to obtain a better understanding of the penetration event, the incremental Eulerian DIC analyses were accumulated in a Lagrangian framework to obtain cumulative displacements. In a Lagrangian reference frame the origin is fixed to the center of each interrogation window, and is assumed to follow the displacements of the interrogation window. The result is that the approximate relative location of a group of particles, initially contained within an interrogation window, is followed as displacements accumulate. For displacement fields with smoothly varying vectors, the Eulerian and Lagrangian methods generally produce similar results. However, when the displacement field is comprised of displacement vectors with large gradients between consecutive images, or within the displacement field itself, Lagrangian predictions of motion and flow can be more useful (Yeung 2002).

Lagrangian analysis requires interpolation of material coordinates using known displacements which, in the case of DIC analysis, are the displacements at the center points of the interrogation windows. The method adopted herein for interpolation of Lagrangian displacements from DIC analysis consisted of employing the Delaunay triangulation described above with nodes defined by the center points of the initial undeformed interrogation windows. Trajectories were obtained using the shape functions used in strain calculations. Details of the algorithms used to produce Lagrangian displacements can be found in Omidvar et al. (2014b). Accumulation of incremental Lagrangian displacements were used in this study to obtain cumulative displacements, presented in the next sections. 


\section{Visualization of Penetration event}

Snapshots of penetration into the embedded plane transparent soil sample are shown in Fig 4 for penetration of blunt and conical nose projectiles, which were propelled with an initial velocity of $21 \mathrm{~m} / \mathrm{s}$ and $22.5 \mathrm{~m} / \mathrm{sec}$, respectively. Impact and initial penetration resulted in cavity formation, which appeared as an opaque region in the images. During the initial stage of penetration, the projectile tip was in contact with the impeding soil, while the afterbody moved in the cavity void. As the penetrator velocity decayed, supercavitation ceased, and the penetrator afterbody was increasingly in contact with the surrounding soil during penetration. Comparison of the blunt and conical nose rods revealed that the opaque region formed in the early stages of penetration of the blunt nose projectile was wider than that of the cone.

During initial embedment and penetration an opaque region formed ahead of the penetrator, with a base diameter greater than that of the penetrator. This opaque region was larger for the blunt nose projectile. The opaque region immediately ahead of the penetrator took the form of an approximate cone in the case of the blunt nose projectile, and persisted ahead of the penetrator during later stages of penetration. However, in the case of the conical projectile, this opaque region did not persist during later stages of penetration. The formation of these opaque regions may be explained by one or more hypotheses, including pressure-induced change in refractive index, cavitation due to tensile hoop strains, and cavitation due to shear-induced dilation.

\subsection{Velocity profiles}

Incremental velocity fields resulting from DIC analysis of successive images are shown in Fig (4c, d). Desaturation may be attributed to dilation-induced cavitation. It can be seen that velocity magnitudes are consistently larger for the blunt projectile compared to the conical nose projectile. In both tests there are nonzero velocity vectors behind the penetration front. These velocity vectors, marking afterflow, are predominantly downward in direction.

Velocity magnitude plots are shown in Fig 5 for blunt and conical nose projectiles, penetrating at depths corresponding to 1D, 2D, 4D, 6D, 8D and 10D. It can be seen that at 1D penetration a hemispherical cavity has formed. This hemispherical region expands during subsequent penetration, as is clear at 2D depth. During later stages of penetration, velocity magnitudes within the hemispherical region decays, and the majority of the velocity profile 
(down to $0.1 \mathrm{~m} / \mathrm{s}$ ) is eventually localized to a region extending approximately 3-5D from the projectile tip. The velocity magnitude beyond this region falls to two orders of magnitude smaller than the impact velocity. Comparison of the blunt and conical nose projectiles reveals that while velocity magnitudes immediatly ahead of the projectile are larger in the blunt projectile, similar velocities are recorded beyond this region for the two projectiles.

In order to further quantify the nature of the soil-projectile interactions, incremental plots of horizontal and vertical velocity were extracted for penetration of the blunt and conical nose projectiles, as shown in Fig 6 and 7. Comparison of horizontal velocity profiles reveals that lateral velocity magnitudes are comparable for the two projectiles, while the zone of influence of the horizontal velocities is larger in the case of the blunt projectiles. In contrast, vertical velocity profiles show that maximum vertical velocities are significantly larger in the case of the blunt projectiles, whereas the zone of influence of the two projectiles is similar. Once the penetrator moves past a given plane, little further lateral displacement occurs in that plane. In contrast to the lateral displacements, contours of vertical displacements reveal that significant vertical after-flow accompanies penetration near the cavity. In other words, behind the advancing penetrator tip, the soil around the cavity has downward flow, particularly within a cylinder of 1-2D from the penetrator.

\subsection{Displacement profiles}

Further characterization of the displacements during various stages of penetration was achieved by observing cumulative Lagrangian lateral and vertical displacements along a fixed plane in the path of the penetrator at a depth of 6D, as shown in Fig 8 for penetration of blunt and conical nose projectiles. For each of the plots in Fig 8, cumulative displacements were obtained from the point of impact to the depth shown, by summing displacement increments from DIC analysis using a Lagrangian framework. In addition to the dynamic penetration tests using conical and blunt nose projectiles, results of the quasi static penetration test is also shown in Fig $8 \mathrm{e}$ and $\mathrm{f}$. Several features can be identified from the cumulative displacement plots:

- In dynamic tests, downward movement of soil ahead of the projectile is significantly larger than the horizontal component. In other words, dynamic penetration produces velocity vectors that deviate from spherical and cylindrical cavity expansion adjacent to the projectile tip. Downward and lateral displacements are more comparable in the case 
of quasi-static penetration, in greater agreement with spherical cavity expansion. For example, in the case of the blunt nose projectile, the ratio of maximum vertical to horizontal cumulative displacement at 6D penetration was approximately 2.7 and 1.5 for dynamic and quasi-static penetration, respectively.

- In dynamic penetration of both conical and blunt projectiles, lateral displacements extend farther away from the projectile trajectory compared to downward movement. Immediately ahead of the projectile, both downward and lateral displacements are larger for the blunt projectile in dynamic penetration. Maximum cumulative downward displacements were 4.7 and $1.8 \mathrm{~mm}$ for the blunt projectile, and 3.7 and $1.2 \mathrm{~mm}$ for the conical nose projectile. However, beyond the immediate region ahead of the projectile, downward and lateral soil movement ahead of the projectile were comparable.

- Comparison of quasi-static and dynamic penetration reveals that the magnitude of maximum lateral displacements adjacent to the projectile is comparable in quasi-static and dynamic penetration. However, the zone of influence of the lateral displacements is significantly larger in the case of dynamic penetration. Accordingly, it can be seen that lateral flow is constrained to a region extending approximately $2 \mathrm{D}$ from the penetrator centerline in the case of quasi-static penetration, whereas notable displacements are observed up to 6D from the projectile trajectory in dynamic penetration. Moreover, while during quasi-static penetration there is little lateral flow beyond approximately 1D ahead of the projectile, dynamic penetration induces notable displacements at up to 3D ahead of the penetrator, for the impact velocity considered in this study. Finally, downward displacements are significantly larger in dynamic penetration compared to quasi-static penetration. The zone of influence of the downward movement is also considerably larger in dynamic penetration, similar to lateral displacements.

- Notable upward flow of soil can be identified in quasi-static penetration as the penetrator approaches a given depth. This upward flow is not observed in the case of dynamic penetration, and displacements are persistently downward for both conical and blunt nose penetrators.

It is noteworthy that in all cases shown, the cumulative horizontal displacements reveal that net lateral flow of soil is significantly lower than that required to accommodate penetration. For example, it can be seen that at $6 \mathrm{D}$ penetration in the case of the blunt projectile, where the penetrator has reached the horizontal profile depth, a maximum net displacement of 1.8 
$\mathrm{mm}$ occurs on either sides of the penetrator, which is significantly lower than the $5 \mathrm{~mm}$ required to accommodate the penetrator. Significant net vertical displacements occur, however, at this depth. Finally, it is also noted that the displacements plotted and calculated in Figs 5-8 exclude displacements occurring in the first $0.1-0.3 \mathrm{D}$ immediately adjacent to the penetrator, particularly during the first 1-3D of penetration, since displacements in this region are masked by the aforementioned opaque region.

\subsection{Shear and volumetric strain}

Displacements obtained from image analysis were used to derive shear and volumetric strain fields following methods described earlier. Incremental shear strains corresponding to penetration increments at 1D, 2D, 4D and 6D penetration depth are shown in Fig 9 for blunt and conical projectiles. It can be seen that the large shear strains occur at the projectile tip for both projectiles. Comparison of the plots of incremental shear strain at different penetration depths for the blunt nose projectile suggests that the opaque region, visible in Fig. 4, which formed ahead of the penetrator during the early stages of penetration may delineate an outer boundary of soil continuously shearing in front of the tip. This body of soil, which travels with the penetrator, forms an apparent extended tip at the penetration front. The formation of a false cone during penetration was investigated by Omidvar et al. (2015b). It is also noteworthy that the large shear strains formed ahead of the penetrator indicate that for the penetration velocities encountered in this study, shear induced flow is an important contributing penetration mechanism. Qualitative differences between the strain fields from blunt and conical nose shaped projectiles can be recognized from Fig 9. Significantly larger shear strains result from incremental penetration of the blunt projectile, compared to the conical projectile. The maximum shear strain over the penetration increments shown is 0.54 for the blunt projectile and 0.19 for the conical projectile. Shear strains extend farther outward from the projectile in the case of the blunt projectile. In both cases, the greatest intensity of shear strain is observed immediately beyond the boundary of the opaque region, while smaller strains occur extending approximately 3D and 2.5D outward radially for blunt and conical projectiles, respectively. Further comparison of shear strains from the two projectile shapes reveals that for the blunt nose greatest shear strains occur below the projectile tip, while in the case of the conical nose, the largest shear strains are observed along the cone walls. 
Further insight into meso scale granular kinematics can be gained from plots of volumetric strain, shown in Fig 10. Regions of significant compression are formed immediately adjacent to the penetrator, beyond the opaque region. Significant compression also continues around the cavity as the penetrator passes a given point. This is consistent with postmortem observations reported by Cooper and Breaux (2010) who reported that a layer of compacted sand forms around the cavity left behind the penetrator. It can also be seen from Fig. 10 that considerable dilation occurs ahead of the penetrator beyond the compression zone, at all stages of penetration. This dilation may be a result of tensile hoop strains developed ahead of the expanding cavity. In fact, the majority of volumetric strains deeper than $0.5-1 \mathrm{D}$ from the penetrator tip are dilative strains, extending up to 3D from the penetration front. Comparison of volumetric strains from blunt and conical shaped projectiles reveals that compressive strains are larger in the case of conical projectile. On the other hand, larger dilation occurs in the blunt projectile, extending farther away from the projectile, compared to the conical nose projectile.

\section{Conclusions}

Results of penetration tests into RI matched transparent soils were presented in this paper. Penetration tests were performed using long rods at impact velocities of approximately 20 $\mathrm{m} / \mathrm{s}$. The following observations were made from the penetration tests:

- Incremental displacement profiles of blunt and conical nose projectiles in transparent crushed fused quartz showed that the displacement patterns were generally comparable, although both vertical and lateral displacements were larger and extended farther outwards from the projectile trajectory in the case of the blunt nose projectile.

- Cumulative displacements over 6D penetration using conical and blunt projectiles revealed that in dynamic penetration, downward movement of soil ahead of the projectile is significantly larger than the horizontal component, whereas downward and lateral displacements are more comparable in the case of quasi-static penetration. For example, in the case of the blunt nose projectile, the ratio of maximum vertical to horizontal cumulative displacement at 6D penetration was approximately 2.7 and 1.5 for dynamic and quasi-static penetration, respectively.

- Comparison of dynamic and quasi-static penetration into transparent soils revealed that the magnitude of cumulative lateral displacements was comparable in the two cases, whereas 
significantly larger cumulative vertical displacements were produced during dynamic penetration. For example, at 6D penetration, net vertical displacements were approximately $90 \%$ larger in the case of dynamic penetration compared to quasi-static penetration, in the case of blunt nose projectile.

- The blunt projectile produced larger shear and volumetric strains compared to the conical projectile (0.54 compared to 0.19 for penetration increments at $1 \mathrm{D}-6 \mathrm{D}$ penetration). The zone of influence of the shear and volumetric strains were also larger in the case of the blunt nose projectile.

- Evidence of shear-induced dilation near the terminal penetration velocity was presented, pointing to the dominance of frictional bearing resistance towards the terminal penetration depth.

\section{Acknowledgements}

The authors gratefully acknowledge the support of the Defense Threat Reduction Agency (DTRA) of the United States, Grant No. HDTRA1-10-1-0049. The help of Jeanne Doreau Malioche in performing tests is greatly appreciated.

\section{References}

Omidvar, M., Chen, C., Iskander, M., 2014b. Image-based Lagrangian analysis of granular kinematics. J. Comput. Civil Eng. http://dx.doi.org/10.1061/(ASCE)CP.1943-5487.0000433, 04014101.

Omidvar et al. 2015 (IJIE PAPER under review).

Cooper, W.L., Breaux, B.A., 2010. Grain fracture in rapid particulate media deformation and a particulate media research roadmap from the PMEE workshops. Int. J. Fract. 162 (1-2), 137-150.

Omidvar, M., Malioche, J.D., Chen, Z., Iskander, M., Bless, S., 2015. Visualizing kinematics of dynamic penetration in granular media using transparent soils. Geotech. Test. J. 38(5), 18p.

Collins, A.L., Addiss, J.W., Walley, S.M., Promratana, K., Bobaru, F., Proud, W.G., Williamson, D.M., 2011. The effect of nose shape on the internal flow fields during ballistic penetration of sand. Int. J. Impact Eng. 38, 951-963.

Borg, J.P., Morrissey, M., Perich, C., Vogler, T., Chhabildas, L., 2013. In situ velocity and stress characterization of a projectile penetrating a sand target: experimental measurements and continuum simulations. Int. J. Impact Eng. 51, 23-35.

Nordstrom K.N., Lim E., Harrington M., and Losert W., 2014. Granular Dynamics During Impact. Phys. Rev. Lett. 112; 228002.

Iskander, M., 2010.Modelilng with Transparent Soils, Visualizing Soil Structure Interaction andMulti Phase Flow, Non-Intrusively. Springer. 
Ezzein, F.M., Bathurst, R.J., 2011. A transparent sand for geotechnical laboratory modeling. ASTM Geotech. Testing J. 34 (6), 1-12.

Cave, A., Roslyakov, S., Iskander, M., Bless, S., 2014. Design and performance of a laboratory pneumatic gun for soil ballistic applications. Exp. Tech. http://dx.doi.org/10.1111/ext.12091.

Liu, J.Y., Iskander, M., 2004. Adaptive cross correlation for imaging displacements in soils. ASCE J. Comput. Civil Eng. 18 (46), 12.

Hild, F., Roux, S., 2006. Digital image correlation: from displacement measurement to identification of elastic properties-a review. Strain 42 (2), 69-80.

Sutton, M.A., Orteu, J.J., Schreier, H.W., 2009. Image Correlation for Shape, Motion, and Deformation Measurements: Basic Concepts, Theory and Applications. Springer, New York.

Pan, B., Qian, K., Xie, H., Asundi, A., 2009. Two dimensional digital image correlation for in plane displacement and strain measurement: a review. Meas. Sci. Technol. 20 (6), 062001.

Tong, W., 2005. An evaluation of digital image correlation criteria for strain mapping applications. Strain 41 (4), 167-175.

Westerweel, J., Dabiri, D., Gharib, M., 1997. The effect of a discrete window offset on the accuracy of cross correlation analysis of PIV recordings. Exp. Fluids 23 (1), 20-28.

Lubliner, J., 2008. Plasticity Theory. Dover Publications, New York.

de Berg, M., Cheong, O., van Kreveld, M., Overmars, M., 2008. Computational Geometry: Algorithms and Applications, third ed. Springer, New York.

Yeung, P.K., 2002. Lagrangian investigations of turbulence. Annu. Rev. Fluid Mech. 34 (1), 115-142. 


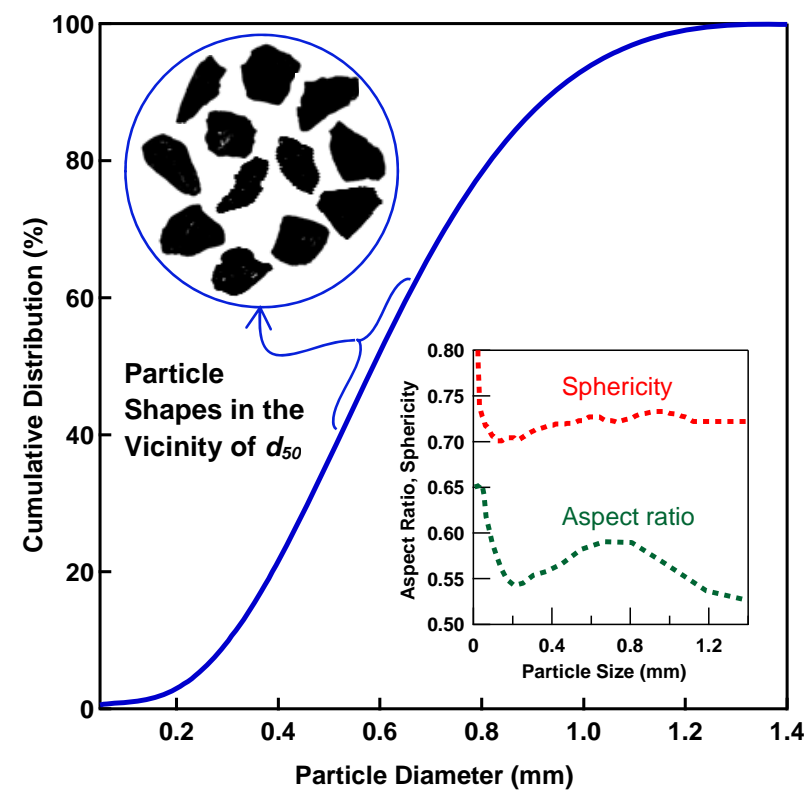

Fig 1: Particle size distribution of dry crushed fused quartz used in this study. Inset shows distribution of sphericity and aspect ratio of particles. 


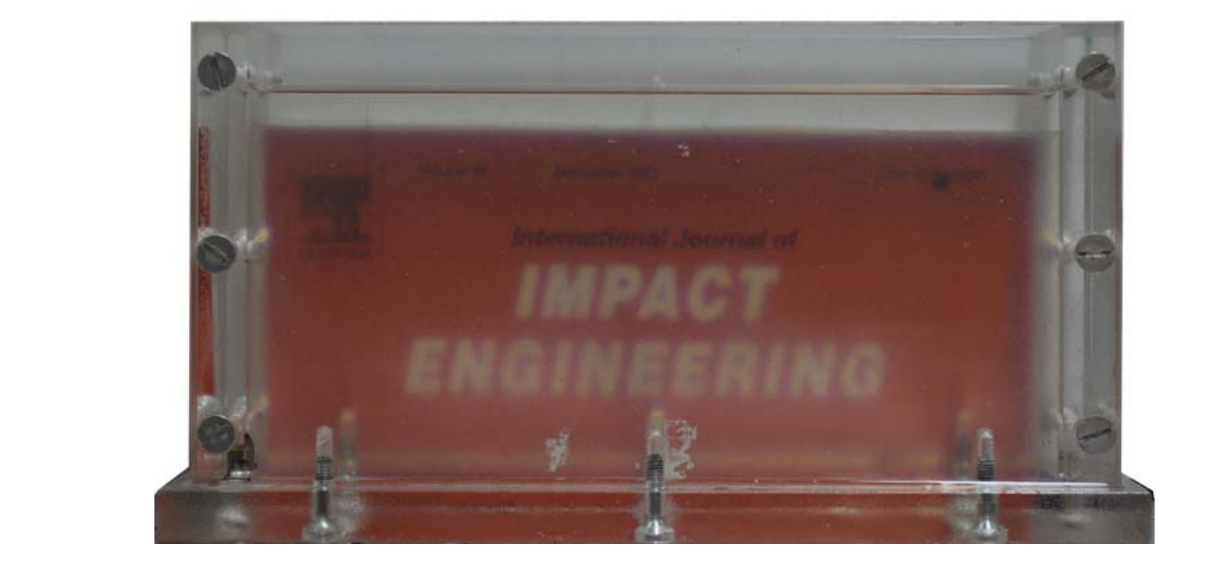

Fig. 2: Journal cover visible through50 mm thick transparent soil model

Figure 2

Fig. 2: Journal cover visible through50 mm thick transparent soil model

(1)

2

Fure

(1)

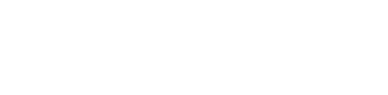

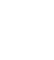




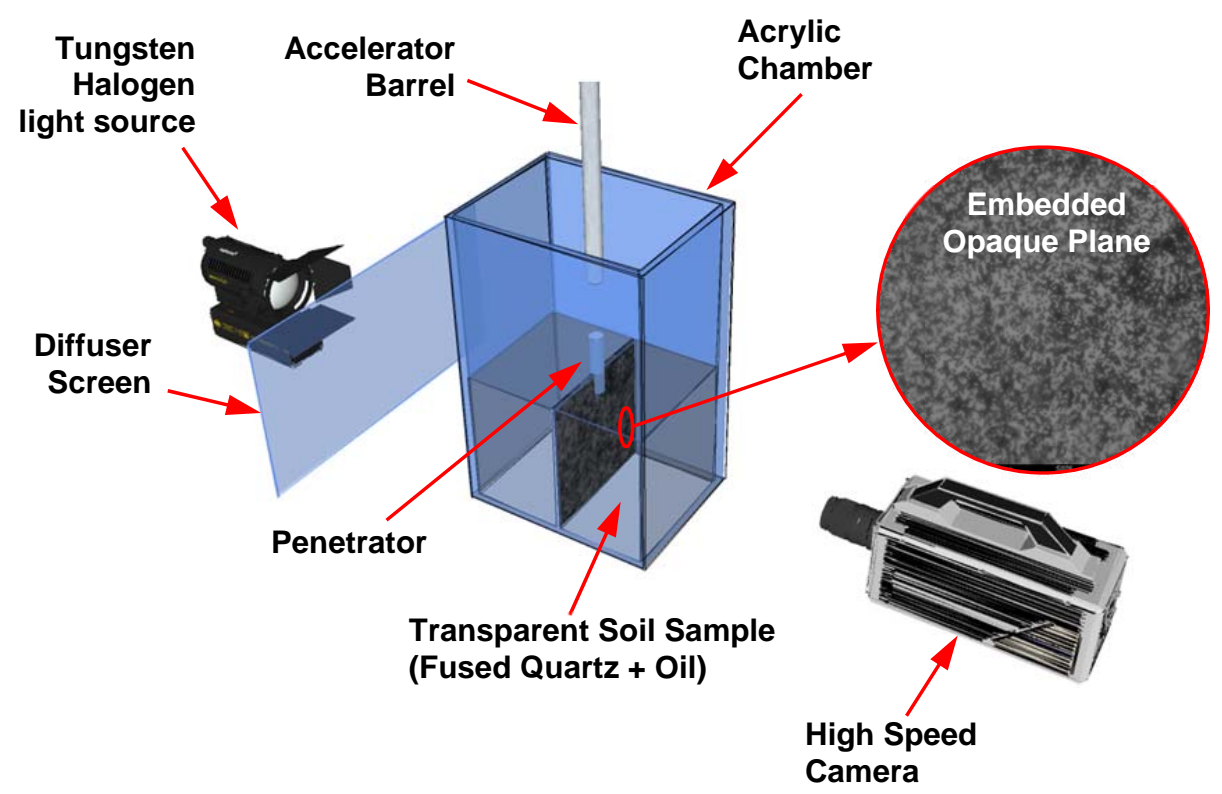

Fig 3: Schematic of setup used to visualize projectile penetration into transparent soil samples 

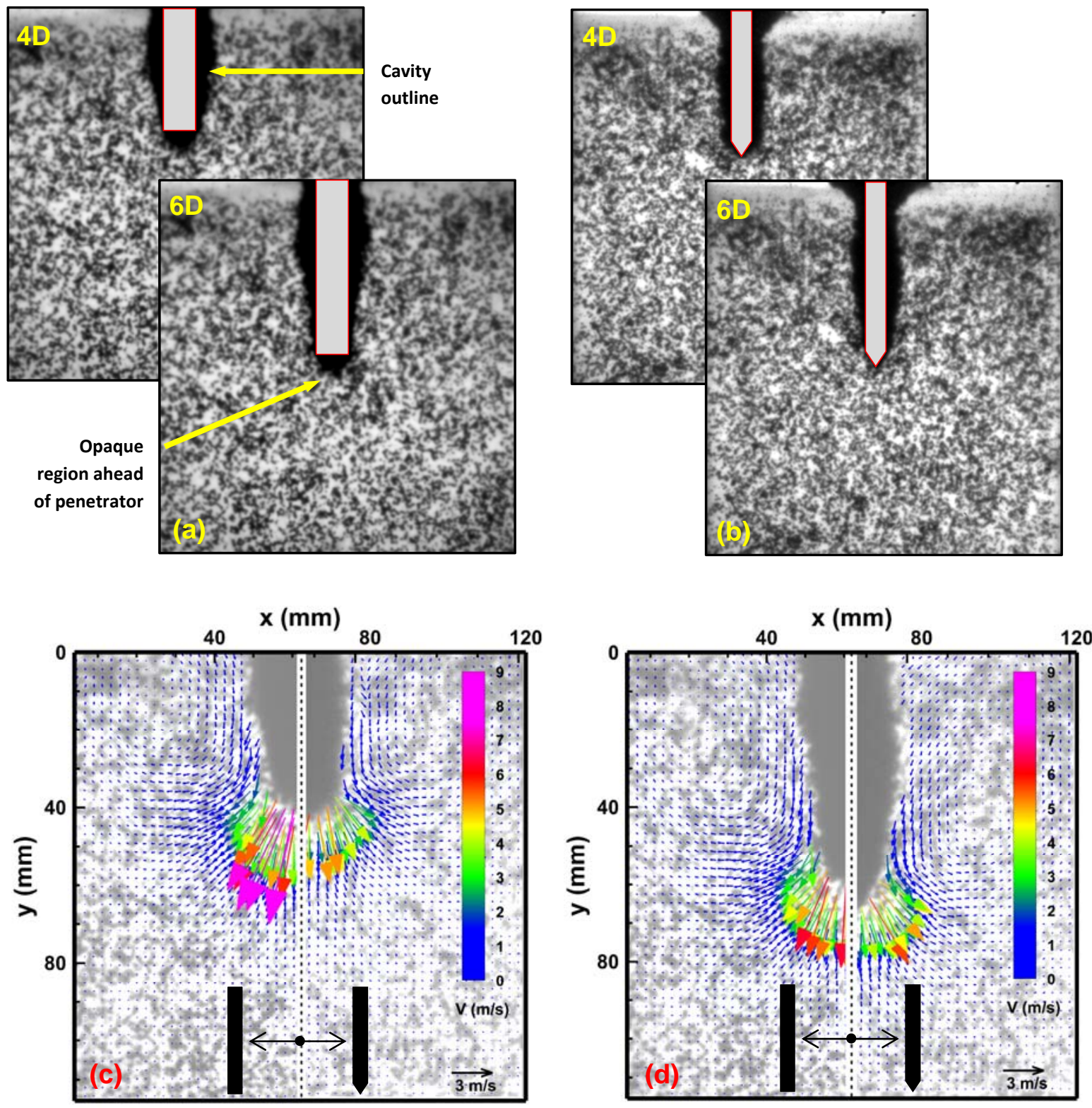

Fig 4: (a, b) Snapshots of penetration of blunt and conical nose projectiles at 4D and 6D penetration depth, and (c, d) corresponding incremental velocity fields resulting from DIC analysis at 4D and 6D penetration depth, for both conical and blunt projectile. 

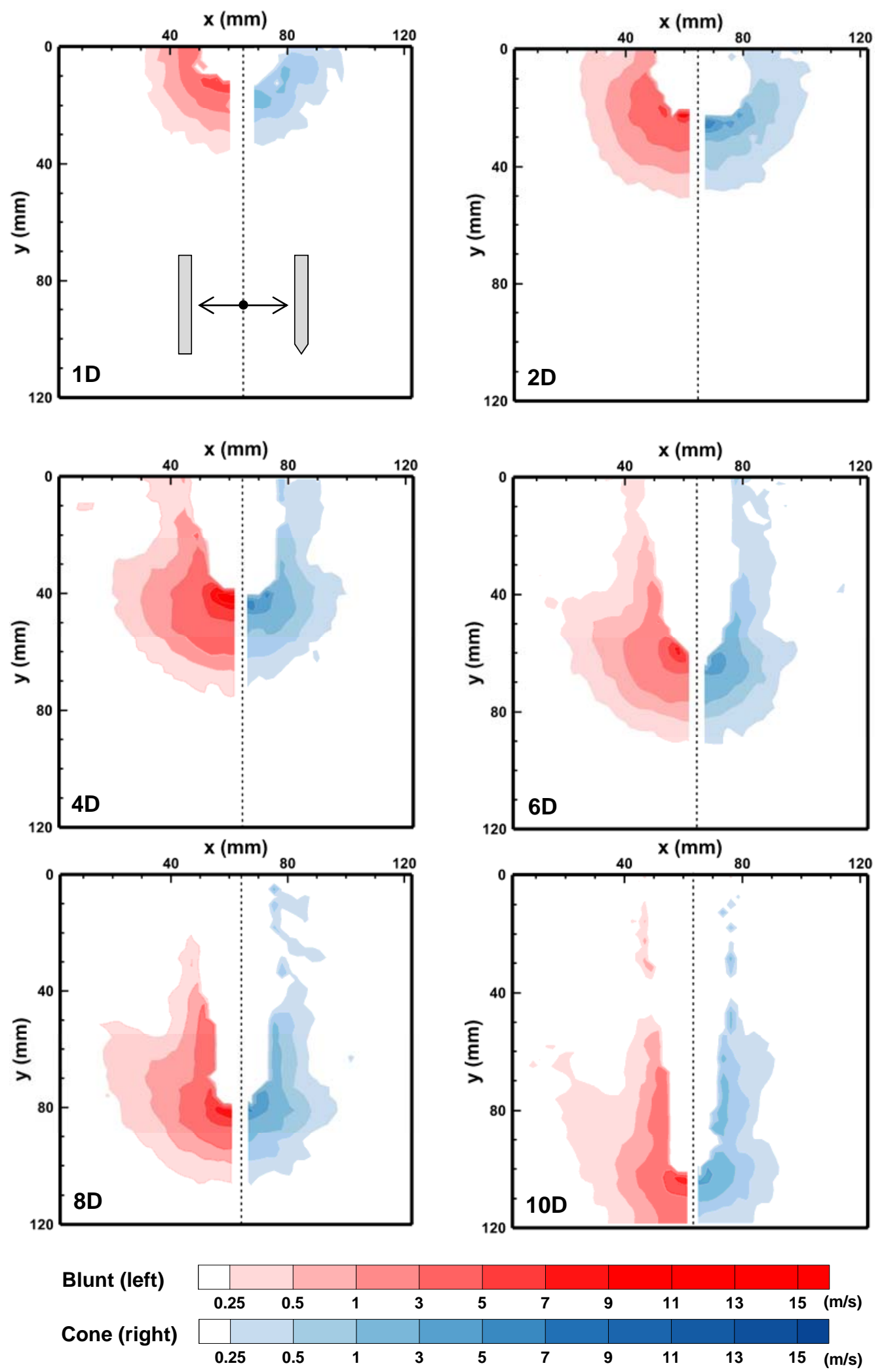

Fig 5: Distribution of velocity magnitude within the soil target induced by blunt and conical nose shaped projectiles for penetration increments at approximately 1D, 2D, 4D, 6D, 8D, and 10D penetration depth. Initial impact velocity $=21 \mathrm{~m} / \mathrm{s}$ and $22.5 \mathrm{~m} / \mathrm{s}$ for blunt and conical projectiles, respectively. 

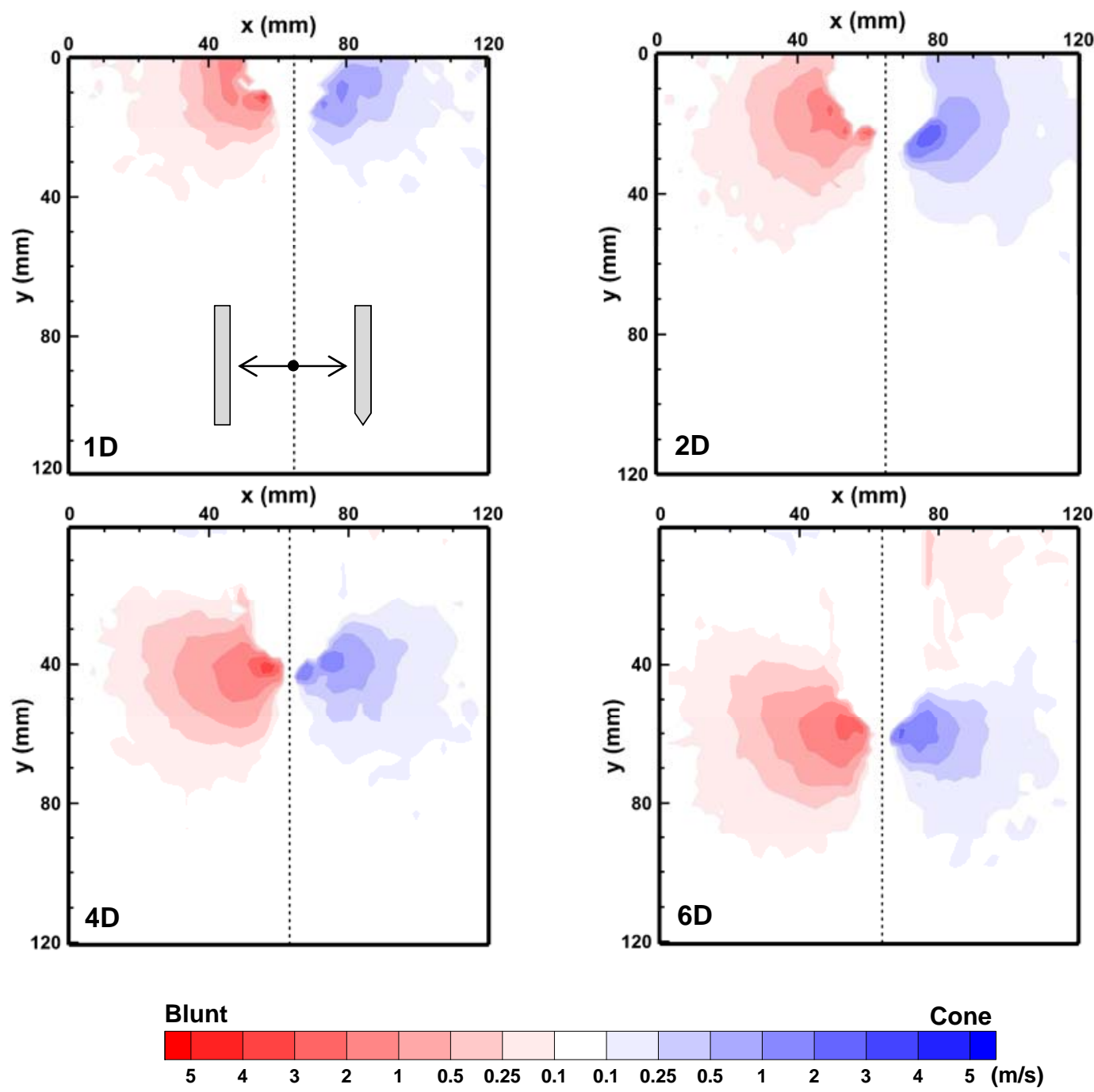

Fig 6: Contours of incremental horizontal velocity field at depths corresponding to penetration depths of 1D, 2D, $4 \mathrm{D}$, and $6 \mathrm{D}$ for penetration of blunt and conical nose projectiles. 

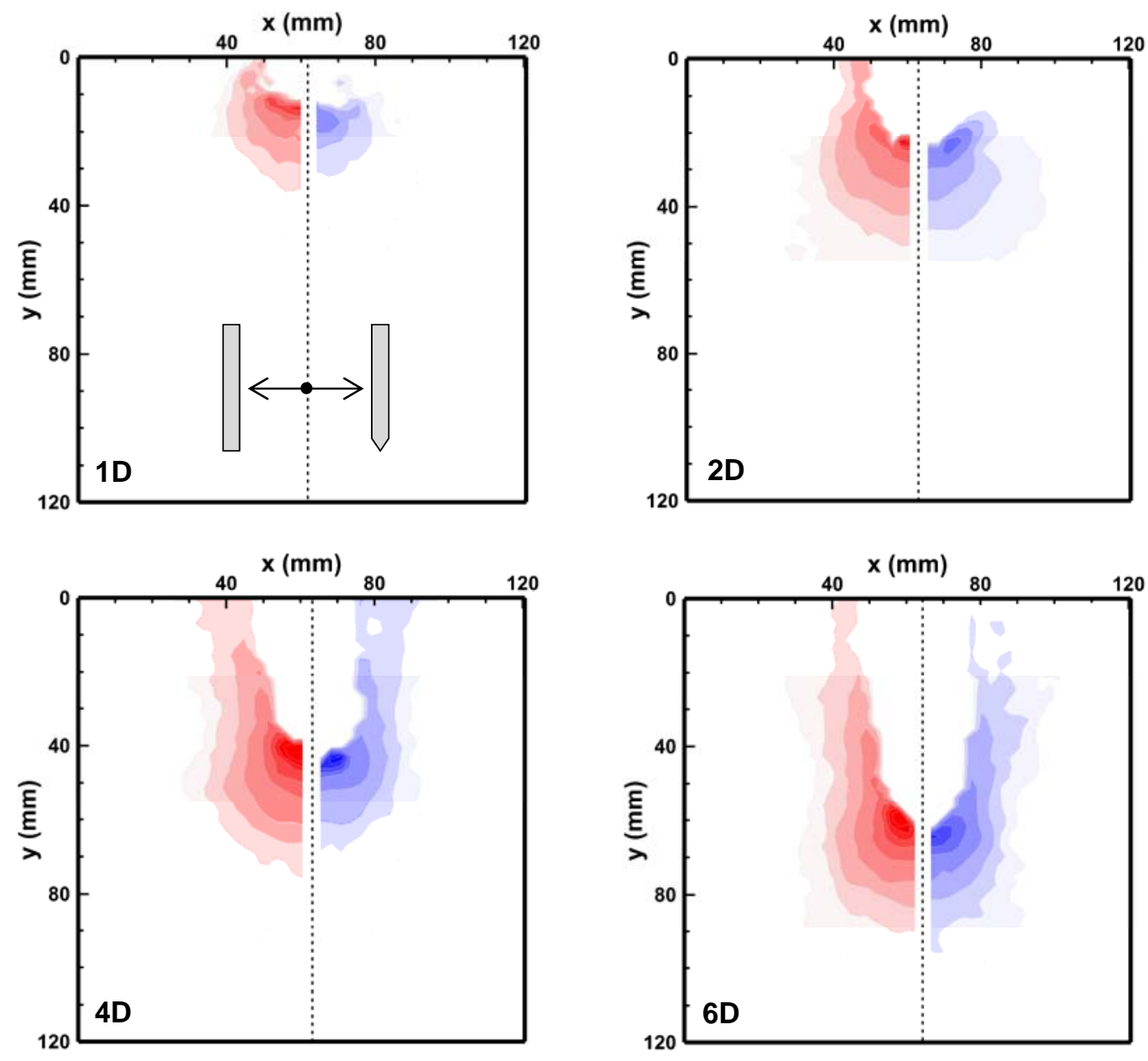

\section{Blunt}

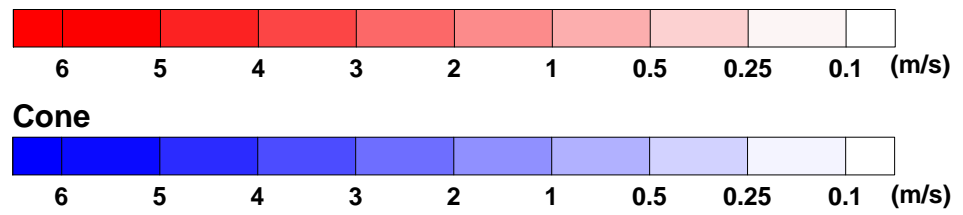

Fig 7: Contours of incremental vertical velocity field at depths corresponding to penetration depths of 1D, 2D, 4D, and 6D for penetration of blunt and conical nose projectiles. Maximum legend value is cutoff value of blunt projectile; maximum values are $8.8 \mathrm{~m} / \mathrm{s}$ and $6.4 \mathrm{~m} / \mathrm{s}$ for blunt and conical nose projectiles, respectively. 

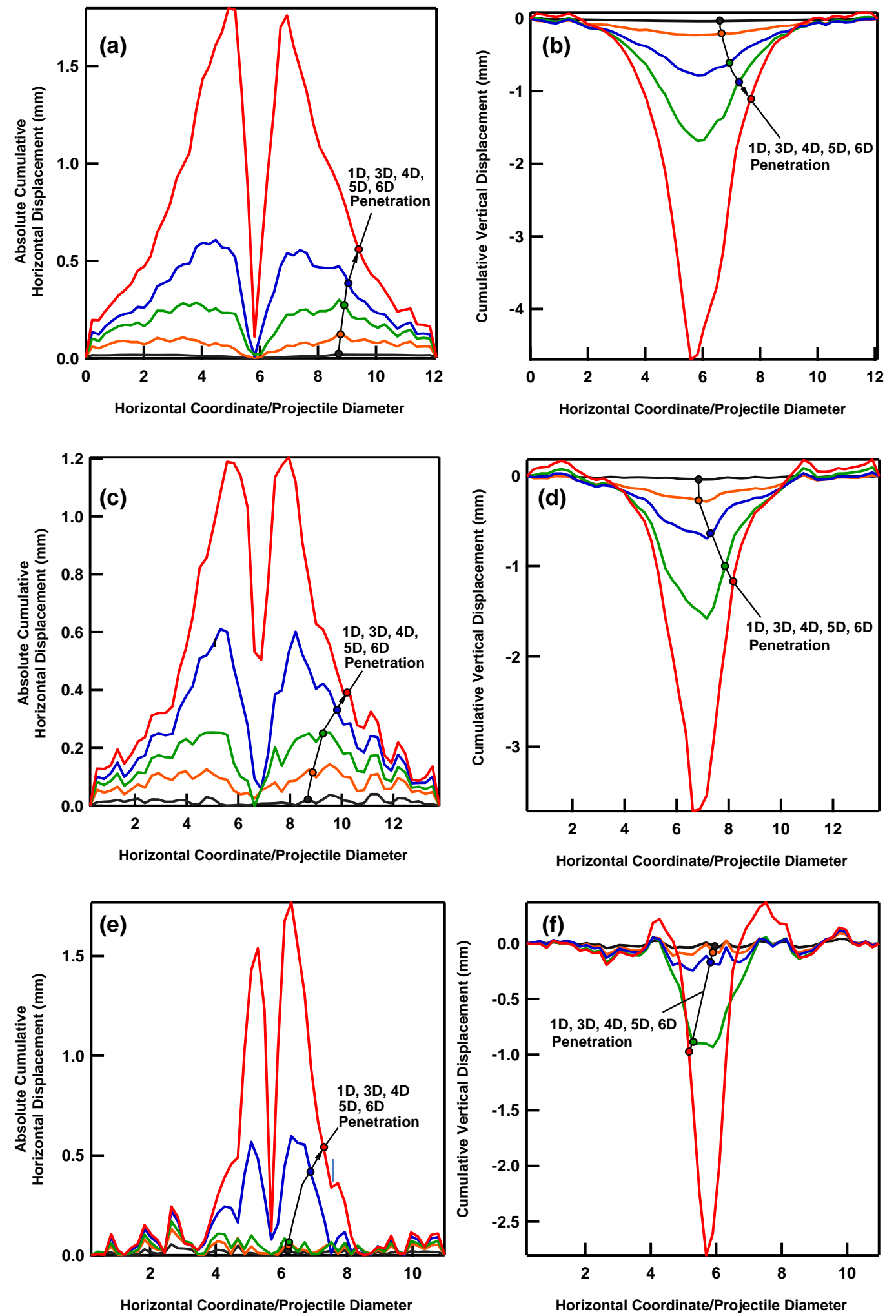

Fig 8: Cumulative horizontal (a, c, e) and vertical (b, d, e) displacements from point of impact to 6D penetration, along a horizontal profile 6D away from the target surface, for blunt (a, b), conical (c, d) projectiles, as well as for quasi static penetration of blunt penetrator $(e, f)$ 

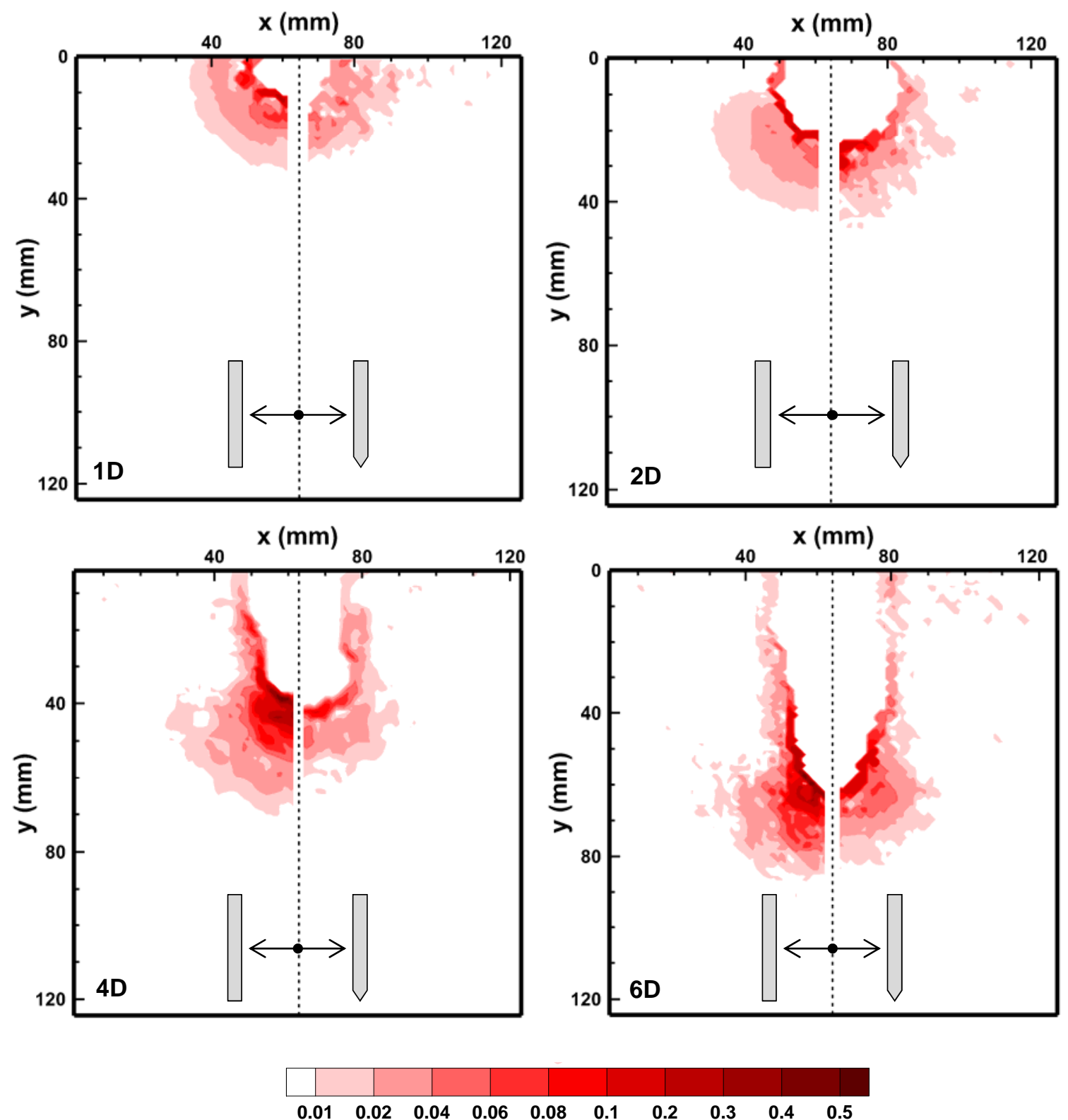

Fig 9: Shear strain from increments of penetration at 1D, 2D, 4D, and 6D penetration depth, for blunt and conical nose projectile. Maximum shear strain: 0.54 and 0.19 for blunt and conical nose projectiles, respectively. 

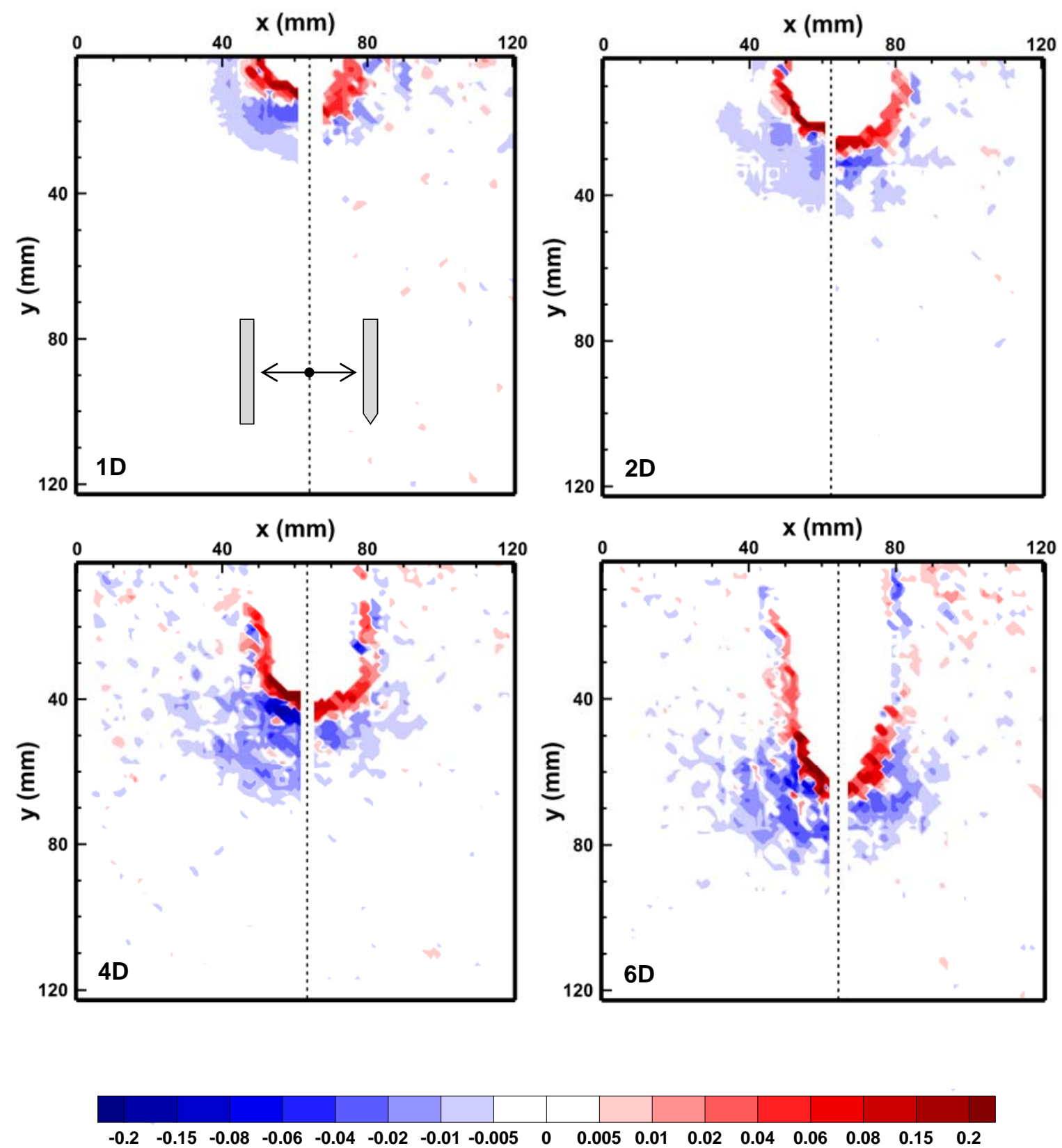

Fig 10: Increments of volumetric strains at 1D, 2D, 4D, and 6D penetration for blunt and conical nose projectiles. Positive and negative values represent compression and dilation, respectively; maximum compression and dilation values shown in legend are cutoff value (compression is positive). 